\title{
Zur Frage der Absorption von Halogenidleuchtstoffen
}

\author{
Von J. Rudolph \\ Aus der OSRAM-Studiengesellschaft, Augsburg \\ (Z. Naturforschg. 18 a, 323-331 [1963] ; eingegangen am 10. Dezember 1962)
}

\begin{abstract}
Es werden die ultravioletten Absorptionsspektren konzentrierter $\mathrm{CaJ}_{2}$ - und einiger $\mathrm{CaBr}_{2}$ - $\mathrm{Lösun}$ gen, die geringe Mengen von $\mathrm{Hg}^{2+}, \mathrm{Tl}^{+}, \mathrm{Sn}^{2+}, \mathrm{Pb}^{2+}, \mathrm{Sb}^{3+}$ und $\mathrm{Bi}^{3+}$ in Form von Halogen-Komplexionen enthalten, bei Zimmertemperatur und bei $-180^{\circ}$ gemessen. Die bei tiefen Temperaturen glasklar einfrierbaren Lösungen zeigen eine erhöhte Auflösung der den Schwermetallkomplexionen zuzuordnenden Absorptionsspektren. Einige dieser Lösungen, insbesondere die mit $\mathrm{Tl}$ und Sn, lumineszieren intensiv. Ihre Lumineszenzspektren werden - ebenso wie die einiger kristalliner Halogenidleuchtstoffe (mit $\mathrm{Sn}, \mathrm{Pb}$ und $\mathrm{Hg}$ ) - ausgemessen.

Der Vergleich der Absorptions- und Lumineszenzeigenschaften der eingefrorenen Komplexsalzlösungen mit denen der entsprechenden Halogenidphosphore zeigt große, auch in Details gehende Ähnlichkeit. An Hand dieses analogen Verhaltens werden Fragen des Absorptionsmechanismus und der Zentrenbeschaffenheit diskutiert.
\end{abstract}

Im Rahmen der Untersuchungen über den Mechanismus der Lumineszenz von Alkalihalogenidphosphoren sind von einer Reihe von Autoren Vergleiche zwischen den Absorptionsspektren der schwermetallaktivierten Alkalihalogenidkristalle einerseits und den Absorptionsspektren der gesättigten, wäßrigen Alkalihalogenidlösungen, in denen geringe Mengen der Aktivatoren in Form von Komplexionen gelöst waren, andererseits herangezogen worden. Auf die große Ähnlichkeit der Absorptionsspektren von Kristall und Lösung ist für die Halogenidsysteme mit geringen $\mathrm{Zusätzen} \mathrm{von} \mathrm{Ag}, \mathrm{Cu}, \mathrm{Tl}$ und $\mathrm{Pb}$ zuerst von Fromherz $^{1,2}$, bei Zusätzen von Sn von Rudolph ${ }^{3}$ und HüNIGER ${ }^{4}$ sowie für Sb-haltige Systeme von SCHLEEdE ${ }^{5}$ hingewiesen worden. Lumineszenzerscheinungen an Schwermetall enthaltenden Halogenidlösungen, zuerst erwähnt von $\mathrm{HiLscH}^{6}$, sind - im Vergleich zu den kristallinen Stoffen - näher untersucht worden von Pringsheim ${ }^{7}$ und speziell an Tlhaltigen Lösungen von $\mathrm{Kato}^{8}$, Makishima und Mitarbeitern ${ }^{9}$ sowie in einer kürzlich erschienenen Arbeit von Brauer ${ }^{10}$.

Auf Grund aller dieser Arbeiten scheint zweifellos ein enger Zusammenhang zwischen den für die Lumineszenz verantwortlichen Zentren in den Halogenidphosphoren und den Schwermetallkomplexionen in den entsprechenden Lösungen zu bestehen. Um

1 H. Fromherz, Z. Phys. 68, 233 [1931].

2 H. Fromherz, Z. Elektrochem. 43, 791 [1937].

3 J. Rudolph, Dissertation, Berlin 1939.

${ }^{4}$ M. Hüniger u. J. Rudolph, Z. Phys. 117, 81 [1940].

5 A. Schleede, Leuchten und Struktur fester Stoffe, R. Oldenbourg-Verlag, München 1943, S. 50.

${ }^{6}$ R. Hrusch, Proc. Phys. Soc., Lond. (Extra Part) 49, 40 [1937]. die Analogie der optischen Erscheinungen zu bekräftigen und in einer Reihe von Details zu erweitern, wurden - wie im folgenden berichtet wird Absorptions- und Lumineszenzeigenschaften verschiedener Schwermetallsalzlösungen mit großem Halogenionenüberschuß bei tiefen Temperaturen untersucht. Es hat sich gezeigt, daß konzentrierte wäßrige Lösungen bestimmter Halogenidsalze, insbesondere die der stark hygroskopischen Halogenide des $\mathrm{Ca}$ bei der Temperatur der flüssigen Luft glasklar einfrierbar sind. Struktur und Eigenschaften scheinen dabei ganz denen eines Glases zu entsprechen insbesondere insofern, als keine definierte Erstarrungstemperatur beobachtbar ist, sondern vielmehr eine allmähliche, sich über einen größeren Temperaturbereich erstreckende Viskositätszunahme der Verfestigung vorausgeht. Solche eingefrorene Lösungen mit Zusätzen von Schwermetallhalogeniden bieten für optische Untersuchungen an den Schwermetallkomplexionen zweierlei Vorteile: 1. Die Struktur der Absorptionsspektren wird gegenüber der der flüssigen Lösungen bei Zimmertemperatur deutlich aufgelöst. 2. Die Lumineszenzhelligkeit kann dabei beträchtlich erhöht werden.

Berichtet wird im einzelnen über die Absorptionsspektren eingefrorener $\mathrm{CaJ}_{2^{-}}$und einiger $\mathrm{CaBr}_{2}$ Lösungen mit Zusätzen der Fremdionen $\mathrm{Tl}^{+}, \mathrm{Hg}^{2+}$,

7 P. Pringsheim u. H. Vogels, Physica 7, 49 [1940].

8 M. Kato, Sci. Pap. Inst. Phys. Chem. Res., Tokyo 42, 35 [1944].

9 H. P. Kallmann u. G. M. Spruch, Luminescence of organic and inorganic materials, Verlag J. Wiley, New York 1962, S. 472 .

10 P. Brauer u. D. Pelte, Z. Naturforschg. 17 a, 875 [1962]. 
$\mathrm{Sn}^{2^{+}}, \mathrm{Pb}^{2^{+}}, \mathrm{Sb}^{3^{+}}$und $\mathrm{Bi}^{3^{+}}$sowie über die Lumineszenzspektren einiger dieser festen Lösungen im Vergleich zur Lumineszenz der entsprechend aktivierten kristallinen Halogenide. Im Anschluß daran werden kurz die beiden Grundvorstellungen über den $\mathrm{Ab}$ sorptionsmechanismus in den Alkalihalogenidphosphoren besprochen: Die Seitzsche Auffassung ${ }^{11} \mathrm{im}$ Sinne einer Anregung der substitutionell im Gitter eingebauten Schwermetallionen und im Vergleich hierzu die Auffassung der Spektren als Sub-RydBERG-Übergänge im Sinne von Transfer-Spektren, wie sie zuerst von $\mathrm{HILSCH}^{6}$ angenommen wurden und neuerdings von Dexter und KNox ${ }^{12}$ als zusätzliche Möglichkeit zur Deutung der Absorptionsspektren von Halogenidphosphoren diskutiert wurden.

\section{Experimentelles}

Die Absorptionsspektren im UV wurden mit Hilfe einer Wasserstofflampe und eines Quarz-Doppelmonochromators von Leitz in Verbindung mit einem Elektronenvervielfacher, die Lumineszenzspektren mit einem Einprismen-Glasmonochromator, ebenfalls von $\mathrm{L}$ e i t z , unter Verwendung von Quecksilber-Hoch- oder -Niederdrucklampen als Erregungslichtquelle gemessen. Als kühlbare Küvette für die Lösungen diente die in Abb. 1 a skizzierte Anordnung, bestehend aus zwei - zwecks Verhinderung des Beschlagens - evakuierten Quarzgefäßen $\mathrm{q}$, die in einem durch die Blende $\mathrm{t}$ (TeflonFolie ca. $50 \mu$ dick) gegebenen Zwischenraum $\mathrm{f}$ die $\mathrm{zu}$ messende Lösung enthielt. Über die metallischen Fassungen $\mathrm{m}$, die in flüssige Luft tauchten, ließen sich die Lösungen einfrieren.
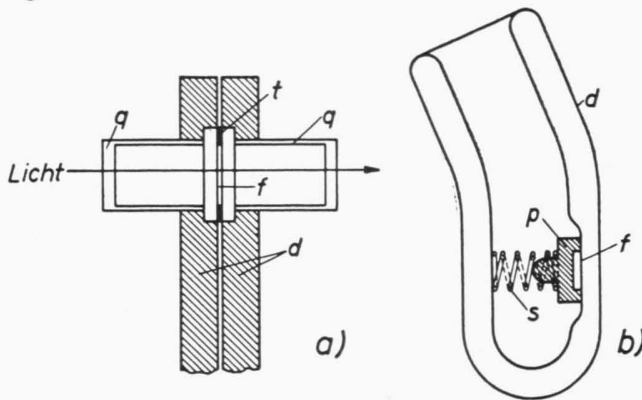

Abb. 1. Anordnung zur Tieftemperaturmessung an Flüssig. keiten a) für Absorption, b) für Emission. q evakuierte Quarzgefäße; t Abstandsblende; f Meßflüssigkeit; m Metallfassungen zur Kühlung; d Quarz-Dewar-Gefäß; p durch Feder s an den Innenmantel gedrückte Metallplatte.

11 F. Seitz, J. Chem. Phys. 6, 150 [1938].

12 R. S. Knox, Phys. Rev. 115, 1095 [1959]; s. auch R.S. Knox u. D. L. Dexter, Phys. Rev. 104, 1245 [1956].

13 H. Fromherz u. W. Menschiк, Z. phys. Chem. B 3, 1 [1929].

14 H. Diamond u. H. Fromherz, Z. phys. Chem. B 9, 289 [1930].
Zur Messung der Lumineszenzspektren der eingefrorenen Lösungen wurde ein Quarz-DEwAR-Gefäß (Abb. $1 \mathrm{~b}$ ) benutzt, das die Lösungen in einer Vertiefung $f$ des Innenmantels, gehalten durch eine federnd an der Wand anliegende ausgesparte Metallplatte p, enthielt.

Die verwendeten Chemikalien waren reinste Stoffe von $\mathrm{M}$ e r ck. Um den Einfluß geringster Verunreinigungen in den konzentrierten $\mathrm{CaJ}_{2}$ - bzw. $\mathrm{CaBr}_{2}$-Lösungen möglichst auszuschalten, wurden die Absorptionen der Lösungen mit den zugesetzten Schwermetallionen jeweils im Vergleich zu denselben, aber zusatzfreien Ca-Halogenidlösungen gemessen. Die Lösungen wurden stets kurz vor der Messung zubereitet und, um Oxydationseffekte $\mathrm{zu}$ vermeiden, so wenig wie möglich mit Luft in Kontakt gebracht. Die Konzentrationen der Lösungen waren in der Größenordnung: Halogenionen ca. $11 \mathrm{Mol} / l$, Schwermetallionen ca. $10^{-3} \mathrm{Mol} / l$.

\section{Meßergebnisse}

\section{Absorptionsspektren der Schwermetallkomplex- salzlösungen}

Es ist insbesondere durch die Arbeiten von FromHERZ und Mitarbeitern ${ }^{13-17}$ bekannt, daß sehr verdünnte Lösungen von Schwermetallhalogeniden, die in der Hauptsache nur hydratisierte Metallionen und Halogenionen enthalten, lediglich im kurzwelligen UV $(<220 \mathrm{~m} \mu)$ absorbieren. Erst bei Zusatz von Halogenionen (als Alkali- oder Erdalkalihalogenide) bilden sich zunehmend mit deren Konzentration im mittleren und langwelligen UV ganz charakteristische Bandensysteme aus, die auf die Assoziation von Schwermetallion und Halogenion zu neutralen Molekülen oder geladenen Komplexmolekülionen der Art $\left[\mathrm{MeHal}_{x}\right]^{y-}$ zurückzuführen sind.

Solche Absorptionsspektren der Komplexionen von $\mathrm{Hg}, \mathrm{Tl}$ und $\mathrm{Pb}$ in konzentrierten $\mathrm{CaJ}_{2}$-Lösungen sind in Abb. $2 \mathrm{a}, \mathrm{b}$ und $\mathrm{c}$ für die flüssigen Lösungen bei Zimmertemperatur und für die bei $-180^{\circ}$ eingefrorenen Lösungen in der Form der Wellenlängenabhängigkeit des Extinktionskoeffizienten

$$
k=(1 / c d) \cdot \log \left(J_{0} / J\right) \quad \text { (in willkürlichen Einheiten) }
$$

wiedergegeben; die Konzentrationen $c$ sind in den Legenden angegeben, die Schichtdicken $d$ waren in der Größe $20-100 \mu$.

15 H. Fromherz u. H. J. Walls, Z. phys. Chem. A 178, 29 [1936].

16 H. Fromherz u. Kun-Hou Lih, Z. phys. Chem. A 167, 103 [1933].

17 H. Fromherz u. Kun-Hou Lih, Z. phys. Chem. A 153, 321 [1931]. 

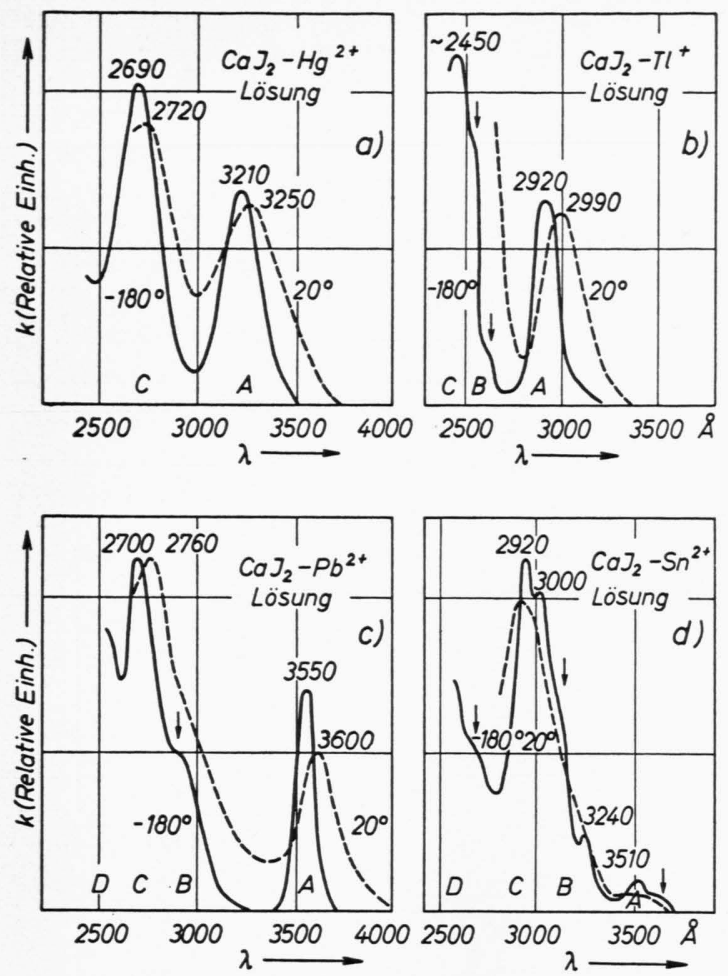

Abb. 2. Absorption konzentrierter $\mathrm{CaJ}_{2}$-Lösungen (5.5 Mol $\left.\mathrm{CaJ}_{2} \cdot 4 \mathrm{H}_{2} \mathrm{O} / l\right)$ mit Zusätzen von (a) $\mathrm{HgJ}_{2}\left(1 \cdot 10^{-3} \mathrm{Mol} / l\right)$; (b) $\mathrm{TlJ}\left(5 \cdot 10^{-3} \mathrm{Mol} / l\right)$; (c) $\mathrm{PbJ}_{2}\left(4 \cdot 10^{-3} \mathrm{Mol} / l\right)$ und (d) $\mathrm{SnJ}_{2}\left(4 \cdot 10^{-3} \mathrm{Mol} / l\right)$.

Das Spektrum der $\mathrm{CaJ}_{2}-\mathrm{Hg}^{2+}$-Lösung (Abb. 2 a) bei Zimmertemperatur, charakterisiert durch die Banden 2720 und $3250 \AA$, stimmt gut mit den von FromHERZ ${ }^{16}$ gemessenen Banden (2670 und $3230 \AA$ ) überein. Bei $-180^{\circ}$ sind die Banden A und $\mathrm{C}^{18}$ schmäler und ein wenig nach kurzen Wellen verschoben. Es tritt indessen - worauf besonders hingewiesen sei - keine Andeutung einer Bandenauf- spaltung oder einer Ausbildung neuer Banden auf. Ein Vergleich mit der Absorption des $\mathrm{Hg}^{2+}$ in Halogenidkristallen ist nicht möglich, da die Herstellung von Alkalihalogenideinkristallen wegen der Flüchtigkeit der Hg-Halogenide schwer möglich ist.

Auch das Absorptionsspektrum des $\mathrm{Tl}^{+}$-Jod-Komplexions (Abb. 2 b) ist durch die zwei ganz ähnlichen Hauptbanden A und $\mathrm{C}$ gekennzeichnet; in der flüssigen Lösung ist die kurzwellige C-Bande wegen des Einsetzens der starken Jodionen-Absorption nicht mehr erfaßbar ebenso wie bei $\mathrm{Fromherz}{ }^{17}$, der nur die langwellige Bande bei $2995 \AA$ in guter Übereinstimmung mit unserem Wert $(2990 \AA)$ messen konnte. Bei der tiefen Temperatur zeigt sich wieder die Verschmälerung und die UV-Verschiebung der Banden, wobei die C-Bande gerade noch dank der Verschmälerung auch der Jodionen-Bande - wenn auch mit einer gewissen Unsicherheit bezüglich Lage und Höhe - erfaßt werden kann. Gleichzeitig zeigt sich die Andeutung neuer schwacher Banden im Gebiet B zwischen und A und C. Ganz das gleiche Bild ist für die Absorptionsspektren der $\mathrm{Tl}^{+}$-aktivierten Jodidphosphore bekannt: besonders mit abnehmender Temperatur tritt zwischen den Hauptbanden A und $\mathrm{C}$ ein neues schwaches Bandensystem, das bei der Temperatur des flüssigen $\mathrm{He}$ z. Tl. aufgelöst werden kann. Tab. 1 zeigt einen Vergleich der Absorptionsbandenlage nach verschiedenen Autoren. Diese Übereinstimmung der einzelnen Banden der in ihrer Form ganz ähnlichen Absorptionsspektren von Komplexlösung und Kristall spricht sehr für das Vorhandensein des gleichen Absorptionszentrums.

Als weiteres Beispiel sei das Absorptionsspektrum einer $\mathrm{CaJ}_{2} \mathrm{~Pb}$-Lösung in $\mathrm{Abb} .2 \mathrm{c}$ wiedergegeben. Die Hauptbanden A und C bei 3600 und $2760 \AA$ (bei Fromherz ${ }^{17} 3595$ bzw. $2720 \AA$ ) rücken beim

\begin{tabular}{|l|c|c|c|c|c|}
\hline \multicolumn{1}{|c|}{ Systeme } & Temperatur & \multicolumn{3}{c|}{ Lage der Banden $(\AA)$} & \multirow{2}{*}{ Autor } \\
& ${ }^{\circ} \mathrm{C}$ & $\mathrm{A}$ & $\mathrm{B}$ & $\mathrm{C}$ & \\
\hline CaJ $_{2}$-Tl-Lösung & 20 & 2990 & - & $<2550$ & \\
CaJ $_{2}$-Tl-Lösung & -180 & 2920 & $2550-2650$ & $\sim 2450$ & \\
KJ-Tl, Einkristall & 20 & 2870 & - & 2360 & V. MEYEREN 19 \\
KJ-Tl, Einkristall & -180 & 2820 & $2400-2500$ & 2330 & Y USTER 20 \\
NaJ-Tl, Einkristall & -180 & 2900 & $2500-2600$ & 2360 & UCHIDA 21,22 \\
\hline
\end{tabular}

Tab. 1.

18 Bezeichnungsweise in Anlehnung an die bei den Halogenidphosphoren häufig verwendete Bezeichnung nach SEITz ${ }^{11}$.

19 W. v. Meyeren, Z. Phys. 81, 321 [1930].

20 P. H. Yuster u. C. J. DelbecQ, J. Chem. Phys. 21, 892 [1953].
21 Y. Uchida u. R. Kato, J. Phys. Soc., Japan 14, 1408 [1959].

22 Die B-Banden werden auf Grund der Konzentrationsabhängigkeit ihrer Intensität teils einzelnen $\mathrm{Tl}$, teils Tl-Paaren zugeordnet. 


\begin{tabular}{|l|r|l|c|c|c|c|}
\hline \multicolumn{1}{|c|}{ System } & $\begin{array}{c}\text { Tempe- } \\
\text { ratur } \\
\end{array}$ & $\begin{array}{c}\text { Messung } \\
\text { in }\end{array}$ & \multicolumn{4}{|c|}{ Lage der Banden } \\
& & A & B & C & D \\
\hline CaJ $_{2}$-Sn-Lösung & -180 & Absorption & $(3600) 3510$ & $3240(3100) 3000$ & 2920 & $(2650)$ \\
CaJ $_{2}$-Sn-poly-kristallin & 20 & Erregung & 36203500 & 325031002970 & 2870 & - \\
KJ-Sn-einkristallin & 20 & Absorption & $(3600) 3500$ & $(3200)(3100) 2970$ & 2870 & - \\
KJ-Sn-poly-kristallin & 20 & Erregung & $(3650) 3510$ & $3220(3100) 2980$ & 2880 & 2650 \\
\hline
\end{tabular}

Tab. 2. Eingeklammerte Banden sind nur durch einen Wendepunkt gekennzeichnet.

Einfrieren der Lösung nach kürzeren Wellenlängen, und eine schwache überlagerte Bande B um 2900 bis $3000 \AA$ kommt deutlicher zum Vorschein. Die Absorption eines entsprechenden einkristallinen $\mathrm{Pb}$ aktivierten Jodidphosphors ist uns nicht bekannt. Indessen ist aus dem Reflexionsspektrum eines polykristallinen $\mathrm{CaJ}_{2}-\mathrm{Pb}$-Phosphors, auf dessen Lumineszenzverhalten weiter unten kurz eingegangen wird, bei $20^{\circ}$ auf zwei starke Absorptionsbanden um 3500 und $2780 \AA \mathrm{zu}$ schließen, die den Banden A und C der Lösung entsprechen.

Ganz ähnlich, aber noch differenzierter ist das $\mathrm{Ab}$ sorptionsspektrum der $\mathrm{CaJ}_{2}$-Sn-Lösung (Abb. $2 \mathrm{~d}$ ). Die stark abgeschwächte A-Bande spaltet bei $-180^{\circ}$ in 2 Banden auf; zwischen der A- und C-Bande erscheinen drei überlagerte Banden (B). An die C-Bande anschließend nach kurzen Wellenlängen erscheint die Andeutung einer weiteren Bande D ${ }^{23}$. Der Vergleich mit den Absorptions- und Erregungsspektren der entsprechenden Sn-aktivierten Jodidphosphore, die vom Verfasser in einer früheren Arbeit untersucht wurden ${ }^{3,4}$, zeigt eine weitgehende Analogie (Tab. 2).

Diese gute Übereinstimmung der Bandensysteme in allen Details, die für die gleiche Beschaffenheit der Absorptionszentren spricht, ist um so bemerkenswerter, als die strukturelle Umgebung des Zentrums jeweils ganz verschieden ist (Flüssigkeit, Glas, Schichtengitter, Steinsalzgitter).

Schließlich seien in Abb. 3 a und b die Absorptionsspektren der Jod-Komplexionen mit den dreiwertigen Metallionen $\mathrm{Sb}^{3^{+}}$und $\mathrm{Bi}^{3^{+}}$wiedergegeben. Das ganz ähnlich aus den Banden A, B und C aufgebaute Spektrum der eingefrorenen Lösungen rückt immer mehr nach langen Wellen, so daß auch in dem im allgemeinen unter der starken Jodionen-Absorption verborgenen kurzwelligen Absorptionsspektren (im Bereich D) Einzelheiten erkennbar werden.

23 Die Bande dürfte den auch bei den Tl-aktivierten Halogenidphosphoren besonders im Erregungsspektrum beobachtbaren D-Banden entsprechen (s. KNox ${ }^{12}$ ).
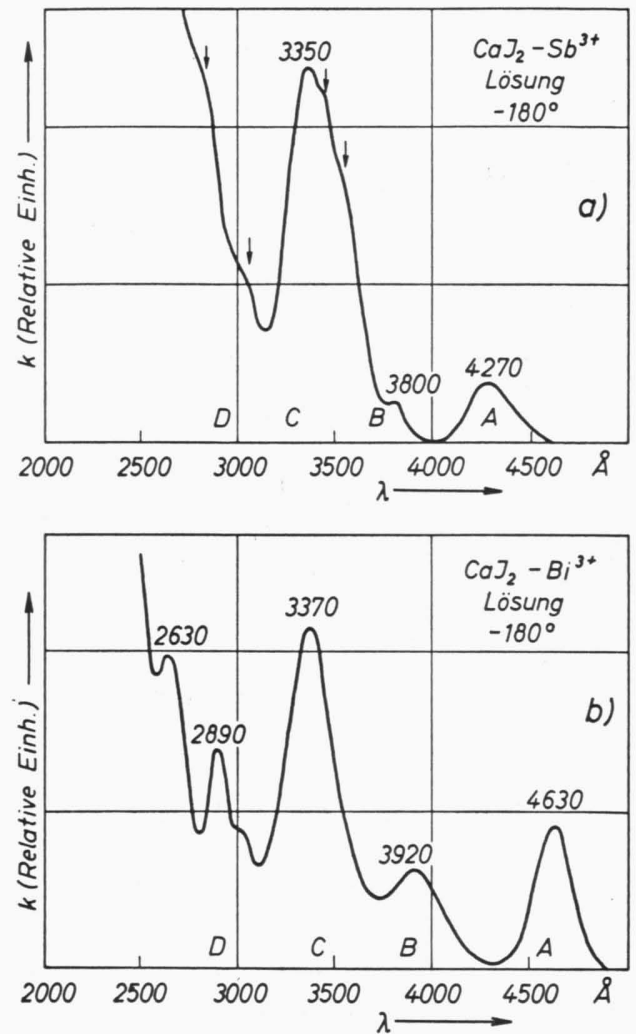

Abb. 3. Absorptionsspektren konzentrierter $\mathrm{CaJ}_{2}$-Lösungen $(5 \cdot 5 \mathrm{Mol} / l)$ mit Zusätzen von (a) $\mathrm{SbJ}_{3}\left(1 \cdot 10^{-2} \mathrm{Mol} / l\right)$ und (b) $\mathrm{BiJ}_{3}\left(5 \cdot 10^{-3} \mathrm{Mol} / l\right)$.

Auch bei den Bromiden haben die Absorptionsspektren der eingefrorenen Komplexionenlösungen die gleiche Gestalt; nur sind sie gegenüber den Jodionen nach kurzen Wellenlängen verschoben. Als Beispiel sind die Spektren von gesättigten $\mathrm{CaBr}_{2}$ Lösungen mit geringen Zusätzen von $\mathrm{Sn}^{2+}$ und von $\mathrm{Tl}^{+}$in Abb. 4 a und c wiedergegeben (der besseren Übersicht wegen ist beim $\mathrm{CaBr}_{2}$-Sn der Logarithmus des Extinktionskoeffizienten aufgetragen). Wieder zeigt sich eine gute Übereinstimmung mit den Bandensystemen der kristallinen Phosphore KBr-Sn bzw. $\mathrm{KBr}-\mathrm{Tl}$ (Tab. 3). 


\begin{tabular}{|c|c|c|c|c|c|c|c|c|}
\hline \multirow{2}{*}{ System } & \multirow{2}{*}{$\begin{array}{l}\text { Tempe- } \\
\text { ratur } \\
{ }^{\circ} \mathrm{C}\end{array}$} & \multirow{2}{*}{$\begin{array}{l}\text { Messung } \\
\text { in }\end{array}$} & \multicolumn{5}{|c|}{ Lage der Banden $\AA$} & \multirow{2}{*}{ Autor } \\
\hline & & & $\mathrm{A}$ & & B & & $\mathrm{C}$ & \\
\hline $\begin{array}{l}\mathrm{CaBr}_{2}-\mathrm{Sn} \text {, gelöst } \\
\mathrm{KBr}-\mathrm{Sn} \text {, kristallin }\end{array}$ & $\begin{array}{r}-180 \\
20\end{array}$ & $\begin{array}{l}\text { Absorption } \\
\text { Absorption } \\
\text { Erregung }\end{array}$ & $\begin{array}{r}3020 \\
(3150) \\
3040 \\
(3170) \\
3050\end{array}$ & $\begin{array}{l}(2750) \\
2770 \\
2770\end{array}$ & $\begin{array}{c}(2650) \\
2650\end{array}$ & $\begin{array}{l}(2600) \\
2570 \\
2570\end{array}$ & $\begin{array}{l}2480 \\
2470 \\
2470\end{array}$ & RUDOLPH ${ }^{3,4}$ \\
\hline $\begin{array}{l}\mathrm{CaBr}_{2}-\mathrm{Tl} \text {, gelöst } \\
\text { NaBr-Tl, kristallin } \\
\text { KBr-Tl, kristallin }\end{array}$ & $\begin{array}{r}-180 \\
20 \\
20 \\
20 \\
20\end{array}$ & $\begin{array}{l}\text { Absorption } \\
\text { Absorption } \\
\text { Absorption } \\
\text { Absorption } \\
\text { Absorption }\end{array}$ & $\begin{array}{l}2600 \\
2670 \\
2610 \\
2600 \\
2610\end{array}$ & $(2450)$ & $\begin{array}{c}- \\
- \\
(2200) \\
(2230)\end{array}$ & $(2200)$ & $\begin{array}{l}2130 \\
2160 \\
2100 \\
2100 \\
2130 \\
2020\end{array}$ & $\begin{array}{l}\text { v. MEYEREN } 19 \\
\text { v. MEYEREN } 19 \\
\text { BÜNGER } 24 \\
\text { LEUTZ }^{25}\end{array}$ \\
\hline
\end{tabular}

Tab. 3.
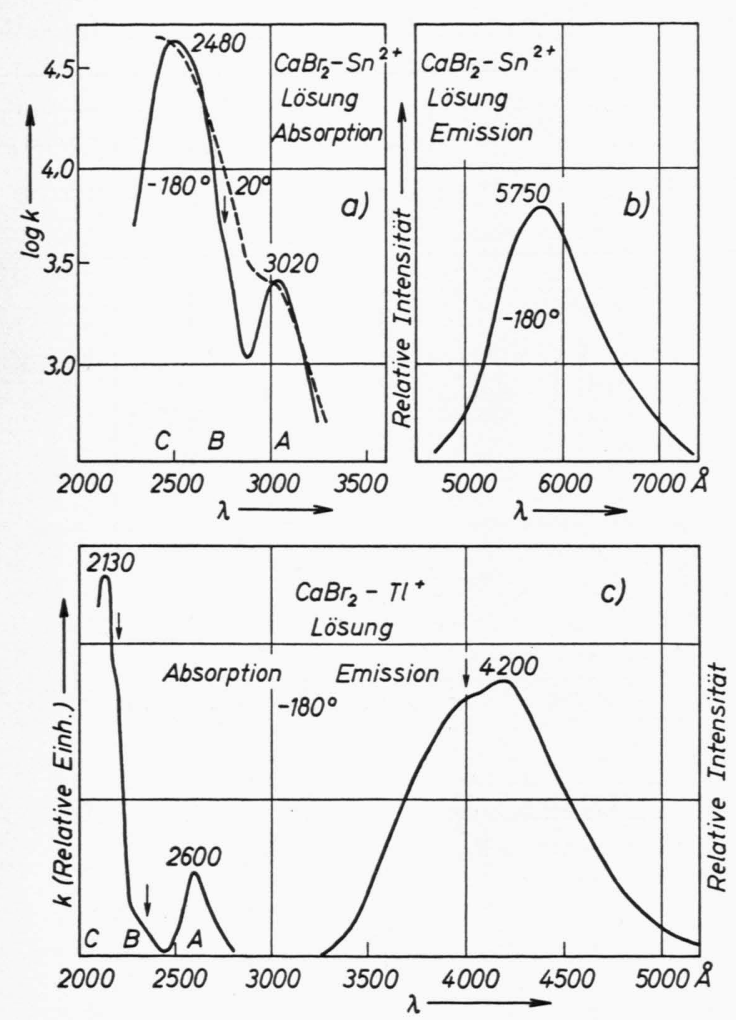

Abb. 4. Absorptions- und Emissionsspektren konzentrierter Lösungen von $\mathrm{CaBr}_{2}(5,8 \mathrm{Mol} / l)$ mit Zusätzen von (a) $\mathrm{SnCl}_{2}$ $\left(5 \cdot 10^{-3} \mathrm{Mol} / l\right)$ und (b) $\mathrm{TlBr}\left(5 \cdot 10^{-3} \mathrm{Mol} / l\right)$. Emission bei Erregung mit einer Quecksilber-Niederdrucklampe.

\section{Lumineszenzspektren der Komplexsalzlösungen}

Wie zuerst $\mathrm{Hilsch}^{6}$, Pringsheim ${ }^{7}$ und Kato ${ }^{8}$ festgestellt haben, lumineszieren die wäßrigen Komplexsalzlösungen einer Reihe von Schwermetallen ( $\mathrm{Tl}$,

24 W. Bünger u. W. Flechsig, Z. Phys. 69, 637 [1931].

25 H. Leurz u. H. Muus, Z. Phys. 155, 531 [1959].
$\mathrm{Pb}, \mathrm{Sn})$ bei Erregung mit UV mehr oder weniger intensiv.

Beim Einfrieren zeigt sich nun häufig eine beträchtliche Steigerung der Lumineszenzhelligkeit, insbesondere bei den Sn-enthaltenden Lösungen, deren Quantenausbeute mit der der hellsten Leuchtstoffe vergleichbar ist. So luminesziert eine glasig eingefrorene Sn-Br-Komplexsalzlösung bei $-180^{\circ}$ hellgelb mit einer Bande um $5750 \AA$ (Abb. 4 b), eine Sn-J-Komplexsalzlösung intensiv orange. Diese Emissionsbande der Komplexsalzlösung bei $6220 \AA$ ist - wie Abb. 5 a zeigt - im Vergleich zur grünen Emission des wasserfreien kristallinen $\mathrm{CaJ}_{2}$-Sn-Phosphors ${ }^{26}$ nach Rot verschoben. Indessen zeigt sich bei diesem kristallinen Phosphor eine schwache langwellige Bande, die im Gebiet der Emission der Komplexsalzlösung liegt. Bemerkenswert ist dabei, daß bei einer frisch hergestellten kristallinen Probe bei Zimmertemperatur zunächst nur die grüne Emission beobachtbar ist. Im Verlauf einer Lagerung an der Luft ensteht mit zunehmender Intensität die langwellige Bande unter Abschwächung der grünen Emission, bis schließlich die Lumineszenz völlig von Grün nach Orange umschlägt. Bei längerer Lagerung an Luft zerfließt schließlich das Salz und die Lumineszenzfähigkeit bei Zimmertemperatur verschwindet ganz. Dieser Effekt hängt offensichtlich mit den stark hygroskopischen Eigenschaften des nach dem Schmelzen zunächst wasserfreien $\mathrm{CaJ}_{2}$ zusammen. Es ist daraus zu schließen, daß durch Feuchtigskeitseinfluß Wassermoleküle im $\mathrm{CaJ}_{2}$-Sn-Phosphor aufgenommen werden und die Struktur des Leuchtzentrums verändern. Über Einzelheiten dieser Beeinflussung der Lumineszenz von Sn-enthaltenden Halogenidsyste-

26 Herstellung durch Schmelzen von $\mathrm{CaJ}_{2}$ mit ca. 0,5 MolProz. Sn. 
men, die vermutlich mit der Bildung von AquoMetallhalogenid-Komplexen zusammenhängt, soll später berichtet werden.

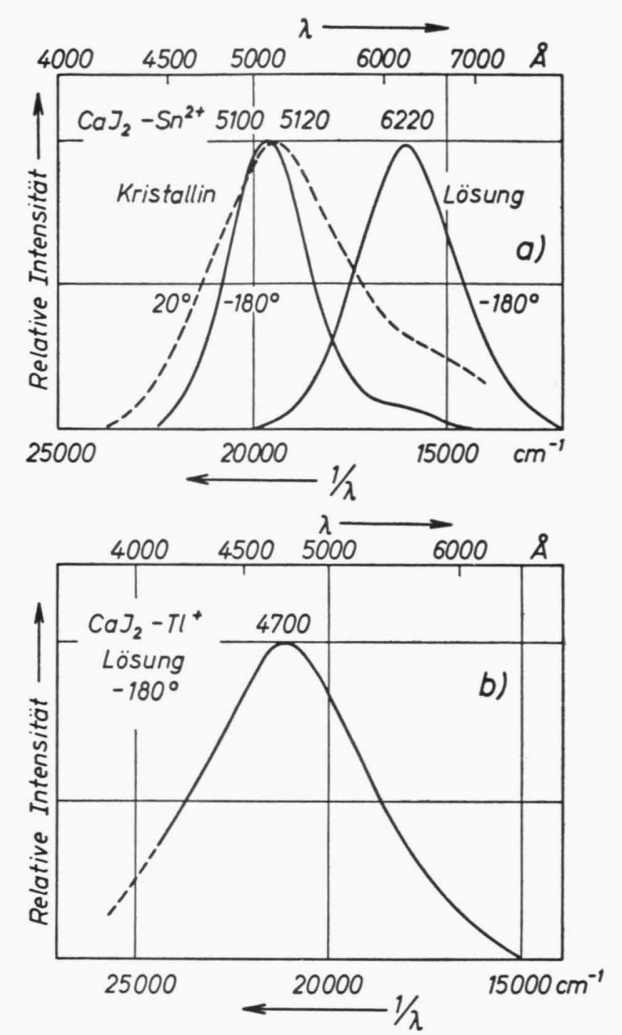

Abb. 5. Lumineszenzspektren der eingefrorenen Lösungen (a) von $\mathrm{CaJ}_{2}-\mathrm{Sn}$ (im Vergleich zur Emission des kristallinen Leuchtstoffes mit 0,5 Mol-Proz. Sn) und (b) von $\mathrm{CaJ}_{2}$-Tl. Konzentration der Lösungen wie bei Abb. 2. Erregung: Quecksilber-Hochdrucklampe.

Eine intensive Lumineszenz tritt auch bei der eingefrorenen $\mathrm{CaJ}_{2}$-Lösung mit $\mathrm{Tl}$ auf; die bläulichweiße Lumineszenz besteht aus einer breiten Bande mit einem Maximum bei $4700 \AA$ (Abb. 5 b), die wiederum langwelliger ist als die Emission der kristallinen Jodid-Phosphore, z. B. bei $-180^{\circ}$ : für NaJ-Tl bei $4250 \AA^{21,27}$ und für KJ-Tl 4270 bis $4300^{19,} 21$.

Die gleichen, offenbar durch die Gegenwart von Wasser-Molekülen verursachten, langwelligen Verschiebungen der Emissionsbanden der eingefrorenen Lösungen gegenüber den kristallinen Systemen sind festzustellen bei der Lumineszenz der Bromide mit $\mathrm{Sn}^{2^{+}}$ bzw. $\mathrm{Tl}^{+}$(Abb. 4b und c) : Gegenüber der $5750 \AA$ Bande der $\mathrm{CaBr}_{2}$-Sn-Lösung emittiert $\mathrm{CaBr}_{2}-\mathrm{Sn}$, 27 W. VAN Sciver, Phys. Rev. 120, 1193 [1960]. wasserfrei, im Blauen bei ca. $4500 \AA, \mathrm{NaBr}$-Sn bei $4600 \AA$ und $\mathrm{KBr}$-Sn bei $5100 \AA^{3,4}$. Kennzeichnend für die $\mathrm{CaBr}_{2}$-Tl-Lösung ist das Auftreten einer Doppelbande (Abb. 4c) ganz ähnlich wie bei der wieder kurzwelliger liegenden Emission der kristallinen Phosphore NaBr-Tl (Maxima bei ca. 2950 und 3080) bzw. KBr-Tl (3190 und $3500 \AA$ ) (v. Meyeren ${ }^{19}$ ). Im übrigen ist eine Verschiebung der bei $-180^{\circ}$ rein blauen Lumineszenz der $\mathrm{CaBr}_{2}$. Tl-Lösung nach Blaugrün bei Temperaturanstieg beobachtbar.

Die eingefrorenen Lösungen von $\mathrm{CaJ}_{2} \mathrm{mit} \mathrm{Hg}$, $\mathrm{Pb}, \mathrm{Sb}$ oder Bi besitzen keine merkliche Lumineszenz im Sichtbaren, obwohl eine Lumineszenzfähigkeit dieser Metalle (mit Ausnahme von Bi) in den kristallinen Jodidphosphoren bekannt ist. So luminesziert KJ-Sb nach $\mathrm{S}_{\text {ChleEde }}{ }^{5}$ rotorange, $\mathrm{CdJ}_{2}-\mathrm{Hg}$ nach KutZelnigG ${ }^{28}$ orange sowie $\mathrm{CaJ}_{2}-\mathrm{Pb}$ blaugrün.
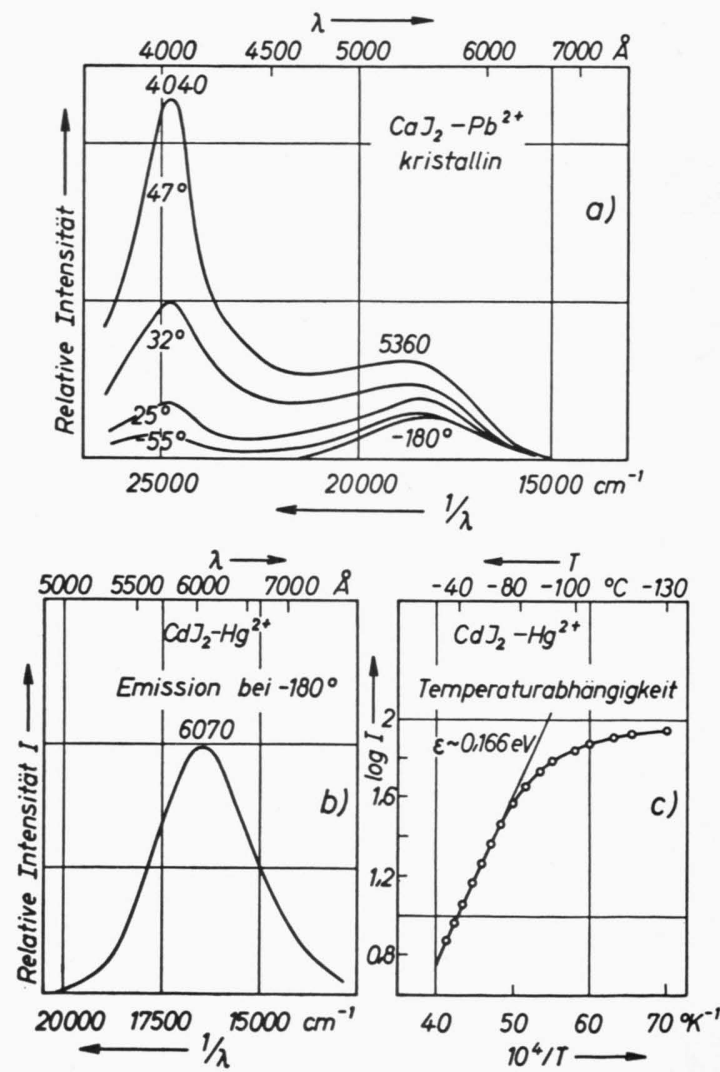

Abb. 6. Lumineszenzspektren der kristallinen Leuchtstoffe (a) $\mathrm{CaJ}_{2}-\mathrm{Pb}(0,05$ Mol-Proz.) bei Temperaturen zwischen $-180^{\circ}$ und $47^{\circ}$ sowie (b) $\mathrm{CdJ}_{2}-\mathrm{Hg}$ (0,1 Mol-Proz.) bei $-180^{\circ}$ und Temperaturabhängigkeit der Intensität. Erregung: Quecksilber-Hochdrucklampe.

28 A. Kutzelnigg, Z. angew. Chem. 49, 267 [1936]. 
Die Emissionsspektren der letzten beiden Phosphore, für die bisher keine Messungen vorliegen, sind zusammen mit ihrem Temperaturverhalten in Abb. 6 wiedergegeben. Über das Lumineszenzverhalten von $\mathrm{CaJ}_{2}-\mathrm{Pb}$ und einiger anderer $\mathrm{Pb}$-aktivierter Phosphore soll in anderem Zusammenhang näher berichtet werden.

\section{Diskusssion}

Durch die erhöhte Auflösung des Absorptionsspektrums in den Komplexionenlösungen bei $\mathrm{Ab}$ kühlung auf die Temperatur der flüssigen Luft wird die Analogie der Komplexionenabsorption und der Absorption der Halogenidphosphore und damit die Annahme gleicher Absorptionszentren ganz offensichtlich verstärkt.

Es erhebt sich die Frage, welche Aussagen sind auf Grund der Kenntnisse über die Beschaffenheit der Komplexionen hinsichtlich der Absorptionszentren und des Mechanismus der Primärabsorption möglich.

Aus den eingehenden Fromherzschen Untersuchungen der Absorption der Lösungen ${ }^{13-17}$ ergibt sich folgender Sachverhalt:

1. Die Absorptionsbanden der hydratisierten Schwermetallionen, gemessen an den kaum zur Ionenassoziation neigenden Chloraten in verdünnter wäßriger Lösung, liegt im allgemeinen sehr kurzwellig: Für $\mathrm{Hg}^{2+}$ bei $<1900 \AA, \mathrm{Tl}^{+}$bei $2140 \AA$, $\mathrm{Pb}^{2+}$ bei $2080 \AA$ und $\mathrm{Sn}^{2^{+}}<1800 \AA$.

2. Mit wachsender Konzentration der Halogenionen in der Lösung steigt die Tendenz zur Assoziation von Metallion und Halogenion zu Molekülen und Molekülkomplexionen, wobei neue Absorptionsbanden auf der langwelligen Seite der Metallionenabsorption entstehen. Dieser Aufbau kann je nach Art des komplexbildenden Metalls diskontinuierlich erfolgen im Sinne des Entstehens neuer, wellenlängenmäßig festgelegter Banden oder kontinuierlich im Sinne eines Aufbaus von Banden, die sich langsam mit zunehmender Halogenionenkonzentration nach langen Wellen bis zu einer Grenzwellenlänge verschieben. Dieses letztere Verhalten hängt mit der konzentrationsbedingten Bildung und der Koexistenz von Komplexionen verschiedenen Asso-

${ }^{29}$ K. Buткоw, Z. Phys. 76, 678 [1931].

30 Kennzeichnend für die Stabilität ist, daß aus der Komplexionenlösung eine Sulfidausfällung trotz der extrem geringen Löslichkeit des $\mathrm{HgS}$ (Löslichkeitsprodukt $\sim 10^{-50}$ ) ziationsgrades zusammen, deren Absorptionsbanden sich überlagern.

Ein Beispiel für den ersten Fall ist das System mit $\mathrm{Hg}^{2+}$. Zunächst entsteht z. B. in der verdünnten Jodidlösung ein Bandensystem aus 2 Banden bei ca. 2200 und $2650 \AA$, das dem undissoziierten $\mathrm{HgJ}_{2}$ zuzuordnen ist; es deckt sich mit dem Absorptionsspektrum des gasförmigen $\mathrm{HgJ}_{2}$ mit Banden bei 2240 und $2660 \AA$, das nach Butкow ${ }^{29}$ ein typisches Dissoziationsspektrum ist im Sinne einer Spaltung in $\mathrm{HgJ}$ $+\mathrm{J}$ bzw. $\mathrm{HgJ}+\mathrm{J}_{\text {(angeregt) }}$; der energetische Abstand der Banden von ca. $1 \mathrm{eV}$ entspricht der Größe der Spin-Bahn-Wechselwirkungsenergie des J-Atoms $(0,94 \mathrm{eV})$. Mit steigender $\mathrm{J}^{-}$-Konzentration wächst ein System mit den beiden Banden bei 2700 und $3200 \AA$ (Abb. 2 a), deren Lage von Anfang an festliegt. Diese Banden sind nach Fromherz dem außerordentlich stabilen Komplexion $\left[\mathrm{HgJ}_{4}\right]^{2-}$ zuzuordnen ${ }^{30}$. Charakteristisch für das $\mathrm{Hg}$ ist dabei - wie aus den Fromherzschen Messungen sowie aus Untersuchungen des RAman-Effektes nach BraunE ${ }^{31}$ folgt - , daß in der Lösung nur die beiden Assoziationsprodukte $\mathrm{HgJ}_{2}$ und $\left[\mathrm{HgJ}_{4}\right]^{2-}$ und keine Zwischenprodukte existieren. Der Absorptionsmechanismus für das $\left[\mathrm{HgJ}_{4}\right]^{2-}$-Spektrum ist nicht bekannt. Da aber eine Anregung von Elektronen in der P-Schale beim valenzgesättigten $\mathrm{Hg}^{2+}$ nicht möglich ist, und eine Anregung von 5d-Elektronen in der teilabgeschlossenen O-Schale aus energetischen Gründen in diesem Spektralgebiet kaum denkbar ist, bleibt wohl nur die Deutung durch die Annahme eines TransferSpektrums im Sinne eines Elektronenüberganges vom Halogenion zum $\mathrm{Hg}^{2^{+}}$-Ion übrig.

Ganz anders liegen die Verhältnisse bei der Komplexionenbildung in den Lösungen mit $\mathrm{Tl}^{+}, \mathrm{Pb}^{2+}$ und $\mathrm{Sn}^{2+}$. Aus den Fromherzschen Untersuchungen ist $\mathrm{zu}$ entnehmen:

1. Es bauen sich mit steigender Halogenionenkonzentration auf der langwelligen Seite der Absorption der hydratisierten Ionen neue Absorptionsbanden auf, wobei sich deren Maxima aber kontinuierlich nach langen Wellen verschieben.

2. Die Halogenionenkonzentrationen, die für das Entstehen der neuen Banden erforderlich sind, sind viel höher als beim Hg. Dieses Verhalten wird dadurch erklärt, daß die koordinativ gesättigten Kom-

nicht erfolgt (F. HeIN, Chemische Koordinationslehre, S. Hirzel-Verlag, Leipzig 1950, S. 543).

31 H. Braune u. G. Engelbrecht, Z. phys. Chem. B 11, 408 [1931]. 
plexe für diese Ionen, und zwar etwa in der Reihe $\mathrm{Pb}, \mathrm{Tl}, \mathrm{Sn}$ instabiler werden und $\mathrm{da} \beta$ verschiedene Zwischenstufen von ungesättigten Komplexen mit im allgemeinen nicht identifizierten Assoziationsprodukten entstehen.

Dieses unterschiedliche Verhalten von $\mathrm{Hg}$ einerseits und $\mathrm{Pb}, \mathrm{Tl}, \mathrm{Sn}$ andererseits scheint auch in den Spektren der eingefrorenen Komplexsalzlösungen zum Ausdruck zu kommen. Beim Hg-Jod-System fehlt jede Andeutung einer B-Bande zwischen den Banden A und C; trotz Untersuchungen an Lösungen der verschiedensten Konzentrationsverhältnisse konnten immer nur die ziemlich symmetrischen Banden $\mathrm{A}$ und $\mathrm{C}$ gefunden werden. Dagegen zeigen sich bei den anderen Metallen in steigender Zahl und Höhe in der Reihe Pb-Tl-Sn die Zwischenbanden im Bereich B; sie sind deutlich bei der tiefen Temperatur, weniger oder gar nicht - wegen der Verwaschenheit der Spektren - bei Zimmertemperatur zu sehen. Man könnte vermuten, daß diese im allgemeinen schwachen Zwischenbanden auf die Koexistenz mehrerer Komplexe verschiedener Sättigungsgrade zurückzuführen sind.

Eine definitive Aussage über den Mechanismus der Absorption der Komplexsalzlösungen ist zur Zeit nicht möglich. Die Analogie der A- und CBandenbeschaffenheit und -lage z. B. der Tl-J-Komplexionenlösung zu der des $\mathrm{Hg}$ würde für ein Transfer-Spektrum sprechen. In Analogie zu den Tl-Phosphoren sollte dagegen bei Gültigkeit der Seitzschen Auffassung eine Anregung von 6s-Elektronen in der P-Schale des $\mathrm{Tl}^{+}$-Ions zu erwarten sein.

SEITz ${ }^{11}$ hat bei der Deutung der Lumineszenz aktivierter Alkalihalogenide dieser Auffassung den Vorzug gegeben vor der Annahme eines TransferSpektrums, wie es von Hilsch angenommen war. Die Gründe hierfür waren: 1. die Invarianz der Absorptionsspektren bei den verschiedenen Halogeniden in der Reihe Chlorid, Bromid, Jodid (Fehlen einer Dublettstruktur mit einem Energieabstand entsprechend der Termtrennung Halogen-Halogen (angeregt) und 2. die aus energetischen Überlegungen abgeleitete Schlußfolgerung, daß solche Transfer-Spektren in den Halogeniden kurzwelliger liegen sollten.

32 P. D. Johnson u. F. E.Williams, Phys. Rev. 117, 964 [1960].

${ }^{33}$ Von N. E. Lushchik ${ }^{34}$ wurde das Modell auch auf die Halogenide mit den zum $\mathrm{Tl}^{+}$isoelektrischen Ionen $\mathrm{Ga}^{+}, \mathrm{In}^{+}$ $\mathrm{Ge}^{2+}, \mathrm{Sn}^{2+}$ und $\mathrm{Pb}^{2+}$ qualitativ angewandt.
Indessen scheint bezüglich Punkt 2. das Beispiel der Hg-Komplexionen-Absorption, die sicher nicht einer Anregung des $\mathrm{Hg}^{2+}$-Ions zuzuschreiben ist, das Auftreten von Transfer-Banden im mittleren UV zuzulassen. Hinsichtlich Punkt 1. sei auf Überlegungen von $\mathrm{K}_{\text {Nox }}{ }^{12}$ verwiesen, wonach das Fehlen einer Dublettstruktur entsprechend der Halogen-Dublettaufspaltung die Möglichkeit des Auftretens von Elektronen-Transfer-Banden nicht unbedingt ausschließt.

Die aus dem Seitzschen Modell entwickelte quantitative Theorie der Lumineszenz der Tl-aktivierten Halogenidphosphore von Johnson und Williams ${ }^{32,33}$ gibt den beobachteten Sachverhalt z. B. beim KCl-Tl weitgehend in bezug auf die Absorptionsbande A und $\mathrm{C}$ und die ultravioletten Emissionsbanden 2470 und $3050 \AA$ wieder. Es bleibt aber das Problem der sicherlich durch Tl-Einzelionen (nicht Ionenpaare) ${ }^{35}$ hervorgerufenen blauen Emịssionsbande (4750 $)$ und deren bevorzugten Erregbarkeit im Gebiet der B-Bande, und es bleibt das Problem der Polarisation der $3050 \AA$-Emissionsbande (im Gegensatz zur $4750 \AA$-Emission) (KLICK ${ }^{36}$ ) für ein Zentrum, das bei Substitutionseinbau des $\mathrm{Tl}^{+}$im Gitter durch Symmetrie gekennzeichnet sein sollte. Und es bleibt schließlich noch die Frage der Deutung der kurzwelligen Absorption im Gebiet D. Diese ist zwar bei den Tl-Phosphoren wegen der Überlappung mit der Grundgitterabsorption nicht direkt erfaßbar, ist aber durch entsprechende Banden im Erregungsspektrum nachgewiesen (s. z. B. KNox ${ }^{12}$ ). Überdies ist dabei anzunehmen, daß es sich um ein kompliziertes, mehrbandiges System handelt, wie die sich zunehmend nach dem Sichtbaren verschiebenden Spektren der Lösungen mit $\mathrm{Sn}, \mathrm{Sb}$ und besonders mit $\mathrm{Bi}$ zeigen.

Die Annahme von Zentren im Sinne von Schwermetall-Halogen-Komplexen verschiedenen Assoziationsgrades, die sich aus der Analogie der Absorptionsspektren von Lösung und kristallinem Phosphor ergibt, gäbe die Möglichkeit zum qualitativen Verständnis der Vielfalt der Erscheinungen in Absorption und Emission der Halogenidphosphore. Besonders sei in diesem Zusammenhang hingewiesen auf die Untersuchungen von BRAUER ${ }^{10}$, aus denen sich eine Abhängigkeit auch der Emissionsbandenlage

34 N. E. Lushснгк, Optics and Spectroscopy 8, 441 [1960].

${ }^{35} \mathrm{Zu}$ folgern aus der Konzentrationsabhängigkeit der Intensität 21,32 .

36 C. C. Klick u. W. D. Compton, J. Phys. Chem. Solids 7, 170 [1958]. 
der Tl-Komplexionenlösungen vom Assoziationsgrad der Komplexe ergeben hat.

Wie nun die aus der Annahme gleicher Zentren in Lösung und Kristall folgende Unterbringung solcher komplexer Moleküle im Gitter vorstellbar ist, läßt sich noch nicht entscheiden. Es wäre lediglich denkbar, daß eine Einlagerung des Aktivators in Versetzungen oder anderen Mikrostörgebieten des Kristalls etwa unter Mitwirkung von Gitterlücken erfolgt, und dadurch auch die Möglichkeit zur Bildung verschiedener Komplexe gegeben ist. In diesem $\mathrm{Zu}$ sammenhang sei auch auf die formelle Analogie zur Lumineszenz von Uranylionen enthaltenden Halogenidleuchtstoffen verwiesen ${ }^{37}$, bei denen ja $\mathrm{Ab}$ -

37 J. E. A. Lys u. W. A. Runciman, Proc. Phys. Soc., Lond. 76, 158 [1960]. sorptions- und Lumineszenzeigenschaften durch die in Halogenid eingebauten Uranylionen oder andere O-haltige Komplexgruppen mit $\mathrm{U}^{6^{+}}$bestimmt sind.

Abschließend sei unter Berücksichtigung der analogen optischen Eigenschaften von Komplexsalzlösungen und Halogenidphosphoren bemerkt, daß zum weiteren Verständnis der Lumineszenz der Halogenidleuchtstoffe sicherlich die Klärung der Frage des Absorptionsmechanismus in Komplexionen und die Ermittlung des Zusammenhanges von Absorption und Komplexkonstitution wesentlich beitragen wird.

Für viele wertvolle Diskussionen und Hinweise möchte ich Herrn Prof. A. Schleede meinen besonderen Dank sagen. Ebenso sei Herrn Prof. E. Krautz bestens gedankt für fördernde Diskussionen bei der Durchführung dieser Arbeit.

\title{
Das Infrarotspektrum des Rubins
}

\author{
Von H. G. HäFELE \\ Aus der OSRAM-Studiengesellschaft Augsburg \\ (Z. Naturforschg. 18 a, 331-335 [1963]; eingegangen am 28. November 1962)
}

\begin{abstract}
Das Infratrotspektrum von ungefärbtem $\alpha$-Korund und von Rubinen mit bis zu $0,5 \% \mathrm{Cr}_{2} \mathrm{O}_{3}$ wurde im Wellenzahlbereich von 300 bis $3000 \mathrm{~cm}^{-1}$ mit polarisiertem Licht untersucht. Aus Reflexionsmessungen bei verschiedenen Einfallswinkeln sowie Absorptionsmessungen wurden die optischen Konstanten $n$ und $\varkappa$ und daraus der Imaginärteil der Dielektrizitätskonstanten $\varepsilon^{\prime \prime}$ ermittelt. Das Schwingungsspektrum ist im untersuchten Konzentrationsbereich unabhängig von der Chromkonzentration. Es ist charakterisiert durch drei starke Banden mit Polarisation parallel zur optischen Achse und fünf Banden senkrecht dazu, die jeweils einem auffallend breiten, kontinuierlichen Untergrund überlagert sind.
\end{abstract}

Im Zusammenhang mit der Verstärkung elektromagnetischer Wellen durch erzwungene Emission (Maser, Laser) hat der Rubin in letzter Zeit sehr großes Interesse gefunden. Bekanntlich handelt es sich dabei um Einkristalle aus $\alpha$-Korund $\mathrm{Al}_{2} \mathrm{O}_{3}$ (Kristallsymmetrie $\mathrm{D}_{3 \mathrm{~d}}^{6}$ ), in welche dreiwertige Chrom-Ionen $\mathrm{Cr}^{3^{+}}$isomorph eingelagert sind. In allen Fällen werden synthetische Kristalle verwendet, die bisher vorwiegend nach dem Verneuil-Verfahren hergestellt werden. Die von den $\mathrm{Cr}^{3^{+}}$-Ionen herrührende Absorption liegt im sichtbaren und

1 S. Sugano u. I. Tsujikawa, J. Phys. Soc., Japan 13, 880, 899 [1958]; dort weitere Literatur.

2 T. H. Maiman u. Mitarb., Phys. Rev. 123, 1151 [1961] ; dort weitere Literatur.

3 A. Linz Jr. u. R. E. Newnham, Phys. Rev. 123, 500 [1961].

4 R. Bauple, A. Gilles, J. Ramond u. B. Vodar, J. Opt. Soc. Amer. 40, 788 [1950].

5 R. W. Kebler, nach Handb. d. Physik, Verlag Springer, Berlin 1958, Bd. 26, S. 793. ultravioletten Bereich und ist ausführlich untersucht worden ${ }^{1,2}$. Die optischen Eigenschaften des Grundgitters, d. h. des ungefärbten $\alpha$-Korunds, sind für verschiedene Wellenlängengebiete bekannt. Die Durchlässigkeit wurde von der UV-Absorptionskante ${ }^{3,4}$ bis ins nahe Infrarot ${ }^{5,6}$ gemessen. Ebenso wurden Brechungsindex ${ }^{7,8}$ und Doppelbrechung ${ }^{8}$ in diesem Bereich untersucht. Im langwelligen Infrarot liegen ältere Reststrahlmessungen ${ }^{9}$ sowie ganz neue Dispersionsmessungen bei Wellenzahlen zwischen 10 und $100 \mathrm{~cm}^{-110}$ bzw. zwischen 30 und $250 \mathrm{~cm}^{-111}$ vor.

${ }^{6}$ U. P. Oppenheim u. U. Even, J. Opt. Soc. Amer. 52, 1078 [1962].

7 I. H. Maltison, F. V. Murphy u. W. S. Rodney, J. Opt. Soc. Amer. 48, 72 [1958].

8 M. A. Jeppesen, J. Opt. Soc. Amer. 48, 629 [1958].

9 M. Parodi, C. R. Acad. Sci., Paris 205, 906 [1937].

10 E. V. Loewenstein, J. Opt. Soc. Amer. 51, 108 [1961].

11 S. Roberts u. D. D. Coon, J. Opt. Soc. Amer. 52, 1023 [1962]. 